\title{
Profile of cancer patients attending a tertiary care center
}

\author{
Unni Xavier A. ${ }^{1}$, Amgiasvasanth A M. ${ }^{2 *}$ \\ DOI: https://doi.org/10.17511/ijmrr.2020.i01.15 \\ 1 Antoney Unni Xavier, Associate Professor, Department of Community Medicine, SUT Academy of Medical Sciences, Thiruvananthapuram, \\ Kerala, India. \\ 2* Amgiasvasanth A M, Assistant Programme Manager, NHM, Office of Deputy Director of Health Service, Nagercoil, Tamil Nadu, India.
}

Introduction: In India, around 555,000 people died of cancer in the year 2010. Cancer is a major cause of morbidity and mortality in developing and developed countries. In many low-income and middle-income countries, including India, most of the population does not have access to a well organized and well-regulated cancer care system. Objective: (1) To study the socio-demographic profile of cancer patients attending tertiary care center.(2) To study the type, site and at what stage cancers are being reported to tertiary care center (3) To study the association of the cancer type with socio-demographic variables. Material and methods: A Hospital-based Cross-Sectional Study carried out from November 2013 to October 2014. Detailed pre-designed and pre-tested proforma is used to collect information on the socio-demographic profile. All diagnosed cancer patients admitted at SDM College of Medical Sciences and Hospital. A total of 381 were studied during the study period. Results: Total of the 381 cancer patients, Males were $154(40.4 \%)$ and females were 227 $(59.6 \%)$, it was found that majority of male, $78(50.6 \%)$ study subjects were in the age group of 60-69 years, in socioeconomic status male subjects were in lower-middle and upper lower class i.e. $40(26 \%)$ female were in upper-middle-class i.e. $70(30.8 \%)$, according to the present study breast cancer $(21.3 \%)$ was most prevalent, followed by CA liver and biliary tract $(14.7 \%)$, followed by lungs $(8.7 \%)$. the male was diagnosed in stage III of disease accounting $67(43.5 \%)$ female patients were diagnosed in stage II 86 (37.9). Conclusion: Breast cancer more common among younger age group and upper SES, Cervical cancer more common among lower SES, Lung cancer in literates'. Liver cancer more common among the older age group and lower SES. So, these groups can be targeted for health education and screening for early diagnosis and treatment.

Keywords: Cancer, Mortality, Morbidity

\section{Corresponding Author}

Amgiasvasanth A M, Assistant Programme Manager, NHM, Office of Deputy Director of Health Service, Nagercoil, Tamil Nadu, India. Email: amgiasvasanth@gmail.com

\section{How to Cite this Article}

Xavier AU, Amgiasvasanth AM. Profile of cancer patients attending a tertiary care center. Int J Med Res Rev. 2020;8(1):97-104.

Available From

https://ijmrr.medresearch.in/index.php/ijmrr/article/ view/1128
To Browse

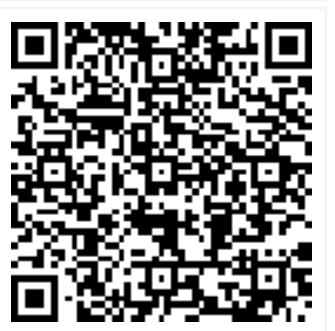

Manuscript Received 2020-01-13

Conflict of Interest No
Review Round 1 2020-01-24

Funding $\mathrm{Nil}$

Review Round 2
2020-01-29
Ethical Approval
Yes

Review Round 2 Yes
Review Round 3

Plagiarism X-checker $6 \%$
Accepted 2020-02-04

Note

(c) 2020 by Antoney Unni Xavier, Amgiasvasanth A M and Published by Siddharth Health Research and Social Welfare Society. This is an Open Access article licensed under a Creative Commons Attribution 4.0 International License https://creativecommons.org/licenses/by/4.0/ unported [CC BY 4.0]. 


\section{Introduction}

Cancer is a major cause of morbidity and mortality in developing and developed countries. In many low-income and middle-income countries, including India, most of the population does not have access to a well organized and well-regulated cancer care system [1].

There were 14.1 million new cancer cases, 8.2 million cancer deaths and 32.6 million people living with cancer (within 5 years of diagnosis) in 2012 worldwide. $57 \%$ ( 8 million) of new cancer cases, $65 \%$ (5.3 million) of the cancer deaths and $48 \%$ (15.6 million) of the 5-year prevalent cancer cases occurred in the fewer developed-regions [2].

The overall age-standardized cancer incidence rate is almost $25 \%$ higher in men than in women, with rates of 205 and 165 per 100,000, respectively. Male incidence rates vary almost five-fold across different regions of the world, with rates ranging from 79 per 100,000 in Western Africa to 365 per 100,000 in Australia/New Zealand (with high rates of prostate cancer representing a significant driver of the latter). There is less variation in female incidence rates (almost three-fold) with rates ranging from 103 per 100,000 in South-Central Asia to 295 per 100,000 in Northern America [2].

In terms of mortality, there is less regional variability than for incidence, the rates being $15 \%$ higher in more developed than in less developed regions in men, and $8 \%$ higher in women. In men, the rates are highest in Central and Eastern Europe (173 per 100,000) and lowest in Western Africa (69). In contrast, the highest rates in women are in Melanesia (119) and Eastern Africa (111), and the lowest in Central America (72) and South-Central (65) Asia [2].

In India 1 million of these new cases and nearly 700 000 of the deaths occurred, which is home to about $17 \%$ of the global population [3].

In 2012, almost 145000 Indian women were diagnosed with breast cancer. Nearly 400000 of those who had reportedly been diagnosed with breast cancer in the previous 5 years were still alive [4].

In India, the five most common cancers in both sexes were

- Breast $(144,937 ; 14.3 \%)$,

- Cervix Uteri $(122,844 ; 12.1 \%)$,
- Lip-Oral $(77,003 ; 7.6)$,

- Lung $(70,275 ; 6.9 \%)$,

- Colorectum $(64,332 ; 6.3 \%)$,

These comprising $47.2 \%$ of the 28 cancers reported. Further, deaths due to these five cancers are 302,124 .

In India, the five most common cancers in men were

- Lung (11.3\%),

- Lip-Oral (11.3\%),

- Stomach (9.1\%),

- Colorectum (7.7\%),

- Oro-pharynx (6.6\%)

These resulting in 219,608 cancers; death due to these cancers were 180,670 , with a five-year prevalence as 235,840 .

In India, the five most common cancers in women were

- Breast (27\%),

- Cervix Uteri (22.9\%),

- Colorectum (5.1\%),

- Ovary (5.0\%),

- Lip-Oral cavity (4.3\%)

A total of 345,191 cancers; death due to these were amounted to 193,664 , with the five-year prevalence as $833,106(5)$, a diagnosis of cancer often leads to catastrophic personal health expenditures. Such expenditures can push entire families below the poverty line [3]. The rapid epidemiological transition has resulted in a shift in the focus from communicable to non-communicable diseases in terms of morbidity and mortality. The cancer profile varies in different parts of the world and an epidemiological study helps us to know the common cancers prevalent in particular segments of a population. However, there is a lack of definitive information regarding hospital-based cancer profile in northern Karnataka. So, the present study is being undertaken with the aim to find out the profile of Cancer patients at SDM College of Medical Sciences and Hospital, Dharwad which is located in Northern Karnataka.

\section{Material and Methods}

Study design: Hospital-Based, Cross-Sectional Study 
Study population: All diagnosed cancer patients admitted in SDMCMSH, Dharwad for treatment

Sample size: All diagnosed cancer patients admitted in SDM College of Medical Sciences and Hospital, during the study period (November 2013 to October 2014) will be enrolled in the study. As per the retrospective data collected from the hospital records, the average number of patients admitted during the last 3 years (who satisfy the present study criteria) accounted for 320 . So, the expected sample size for the present study is estimated to be around 300 .

Sample population: Cancer cases admitted to the hospital for the duration of more than 24 hours and meeting study criteria. A total of 381 were studied during the study period.

Study instrument: Pretested, semi-structured questionnaire was administered by the investigator which relied on ICD-10 for classifying the type of cancer

Study duration: One year [1st November 201330th October 2014]

Study setting: Sri Dharmasthala Manjunatheshwara College of Medical Sciences and Hospital is a tertiary care hospital at Dharwad, Karnataka. The hospital serves as an important reference center for people in and around the district of Hubli-Dharwad.

Ethical clearance: Obtained from the Institutional Ethics Committee, SDMCMS, and $\mathrm{H}$, Dharwad on 8th November 2013

Reference number: SDMIEC: 123: 2013

\section{Results}

It was observed that, regarding the gender-wise distribution of 381 study subjects. Males were 154 (40.4\%) and females were 227 (59.6\%). An agewise distribution of study subjects, it was found that majority of males, $78(50.6 \%)$ study subjects were in the age group of 60-69 years followed by 22 $(14.3 \%)$ in $50-59$ years, $20(13.0 \%)$ in $70-79$ years, and $17(11 \%)$ in $40-49$ years age group.

In female group majority 64 (28.2\%) study subjects were in $50-59$ years followed by $47(20.7 \%)$ in 40 49 years, $40(17.6 \%)$ in 60-69 years, and 30 $(13.2 \%)$ in $30-39$ years age group. In males 78 $(50.6 \%)$ study subjects were in 60-69 years age group which is more when compared to other age
Groups. Majority of study subjects were Hindus i.e. $336(88.2 \%)$ followed by Muslims 34 (8.9\%) Christians and other religions were $11(2.9 \%)$

The majority of study subjects were illiterates i.e. $123(32.3 \%) .50(32.5 \%)$ in males and $73(32.2 \%)$ in females were illiterates. Primary school 87 $(22.8 \%)$, followed by both high school and graduate $63(16.5 \%)$.

The majority of female study subjects were housewives i.e. $115 \quad(50.7 \%)$ followed by agriculturist $47(20.7 \%)$ followed by laborer 27 $(11.9 \%)$. In the male's majority were agriculturist $43(27.9 \%)$ followed by those who were unemployed $29(18.8 \%)$.

According to socioeconomic status majority of study subject males were in lower-middle and upper lower class i.e. $40(26 \%)$ followed by upper class 81 (24.8\%) followed by upper-middle-class 27 (17.5\%) and in female group majority of study subjects were in upper-middle-class i.e. $70(30.8 \%)$ followed by lower-middle-class subjects $54(23.8 \%)$ followed by upper lower class $49(21.6 \%)$. The present study shows that $239(62.7 \%)$ of study subjects were belonging to the nuclear family in both groups. In male group $106(68.8 \%)$ study subjects belonged to the joint family which was more compared to urban area $81(24.8 \%)$ (Table 1$)$.

Table-1: Socio-demographic profile of cancer patients.

\begin{tabular}{|c|c|c|c|c|}
\hline \multirow{9}{*}{ Age } & & Male No. (\%) & Female No. (\%) & Total No. (\%) \\
\hline & 19-29 & $4(2.6)$ & $20(8.8)$ & $24(6.2)$ \\
\hline & 30-39 & $5(3.2)$ & $30(13.2)$ & 35 (9.18) \\
\hline & 40-49 & $17(11.0)$ & $47(20.7)$ & 64 (16.7) \\
\hline & 50-59 & $22(14.3)$ & 64 (28.2) & $86(22.5)$ \\
\hline & $60-69$ & 78 (50.6) & $40(17.6)$ & $118(30.9)$ \\
\hline & 70-79 & $20(13.0)$ & $18(7.9)$ & $38(9.9)$ \\
\hline & $>80$ & $8(5.2)$ & $8(3.5)$ & $16(4.1)$ \\
\hline & Total & $154(100)$ & $227(100)$ & $381(100)$ \\
\hline Sex & & $154(100)$ & $227(100)$ & $381(100)$ \\
\hline \multirow{7}{*}{ Education } & Illiterate & $50(32.5)$ & 73 (32.2) & $123(32.2)$ \\
\hline & Primary School & 38 (24.7) & 49 (21.6) & $87(22.8)$ \\
\hline & High School & $27(17.5)$ & $36(15.9)$ & $63(16.5)$ \\
\hline & PUC / Secondary & $17(11.0)$ & $14(6.2)$ & $31(8.1)$ \\
\hline & Graduate & $18(11.7)$ & 45 (19.8) & $63(16.5)$ \\
\hline & Postgraduate & $4(2.6)$ & $10(4.4)$ & $14(3.67)$ \\
\hline & Total & $154(100)$ & $227(100)$ & $381(100)$ \\
\hline
\end{tabular}




\begin{tabular}{|c|c|c|c|c|}
\hline \multirow{9}{*}{ Occupation } & Unemployed & $\begin{array}{l}29 \\
(18.8)\end{array}$ & 0 & $\begin{array}{l}29 \\
(7.6)\end{array}$ \\
\hline & Agriculturist & $\begin{array}{l}43 \\
(27.9)\end{array}$ & $\begin{array}{l}47 \\
(20.7)\end{array}$ & $\begin{array}{l}90 \\
(23.6)\end{array}$ \\
\hline & Laborer & $\begin{array}{l}18 \\
(11.7)\end{array}$ & $\begin{array}{l}27 \\
(11.9)\end{array}$ & $\begin{array}{l}45 \\
(11.8)\end{array}$ \\
\hline & $\begin{array}{l}\text { Housewife/une } \\
\text { mployed }\end{array}$ & 0 & $\begin{array}{l}115 \\
(50.7)\end{array}$ & $\begin{array}{l}115 \\
(30.1)\end{array}$ \\
\hline & Businessman & $\begin{array}{l}25 \\
(16.2)\end{array}$ & $2(0.9)$ & $\begin{array}{l}27 \\
(7.08)\end{array}$ \\
\hline & Student & $3(1.9)$ & $\begin{array}{l}10 \\
(4.4)\end{array}$ & $\left.\begin{array}{l}13 \\
(3.41)\end{array}\right)$ \\
\hline & $\begin{array}{l}\text { Employee in } \\
\text { service }\end{array}$ & $\begin{array}{l}15 \\
(9.7)\end{array}$ & $\begin{array}{l}18 \\
(7.9)\end{array}$ & $\begin{array}{l}33 \\
(8.6)\end{array}$ \\
\hline & Others & $\begin{array}{l}21 \\
(13.6)\end{array}$ & $8(3.5)$ & $\begin{array}{l}29 \\
(7.6)\end{array}$ \\
\hline & Total & $\left.\begin{array}{l}154 \\
(100)\end{array}\right)$ & $\begin{array}{l}227 \\
(100)\end{array}$ & $\left.\begin{array}{l}381 \\
(100)\end{array}\right)$ \\
\hline \multirow{6}{*}{$\begin{array}{l}\text { Socio economic classification } \\
\text { (according to BG Prasad) }\end{array}$} & Class I & $\begin{array}{l}33 \\
(21.4)\end{array}$ & $\begin{array}{l}43 \\
(18.9)\end{array}$ & $\begin{array}{l}76 \\
(20)\end{array}$ \\
\hline & Class II & $\begin{array}{l}27 \\
(17.5)\end{array}$ & $\begin{array}{l}70 \\
(30.8)\end{array}$ & $\begin{array}{l}97 \\
(25.4)\end{array}$ \\
\hline & Class III & $\begin{array}{l}40 \\
(26.0)\end{array}$ & $\begin{array}{l}54 \\
(23.8)\end{array}$ & \begin{tabular}{|l}
94 \\
$(24.67$ \\
)
\end{tabular} \\
\hline & Class IV & $\begin{array}{l}40 \\
(26.0)\end{array}$ & $\begin{array}{l}49 \\
(21.6)\end{array}$ & $\begin{array}{l}89 \\
(23.3)\end{array}$ \\
\hline & Class V & $\begin{array}{l}14 \\
(9.1)\end{array}$ & $\begin{array}{l}11 \\
(4.8)\end{array}$ & $\begin{array}{l}25 \\
(6.56)\end{array}$ \\
\hline & Total & $\begin{array}{l}154 \\
(100)\end{array}$ & $\begin{array}{l}227 \\
(100)\end{array}$ & $\begin{array}{l}381 \\
(100)\end{array}$ \\
\hline \multirow{5}{*}{ Religion } & Hindu & $\begin{array}{l}137 \\
(89.0)\end{array}$ & $\begin{array}{l}199 \\
(87.7)\end{array}$ & $\begin{array}{l}336 \\
(88.1)\end{array}$ \\
\hline & Muslim & $\begin{array}{l}14 \\
(9.1)\end{array}$ & $\begin{array}{l}20 \\
(8.8)\end{array}$ & $\begin{array}{l}34 \\
(8.9)\end{array}$ \\
\hline & Christian & $0(0)$ & $2(.9)$ & $2(0.5)$ \\
\hline & others & $3(1.9)$ & $6(2.6)$ & 9 \\
\hline & Total & $\begin{array}{l}154 \\
(100)\end{array}$ & $\begin{array}{l}227 \\
(100)\end{array}$ & $\begin{array}{l}381 \\
(100)\end{array}$ \\
\hline \multirow{6}{*}{ Marital } & Married & $\begin{array}{l}119 \\
(77.3)\end{array}$ & $\begin{array}{l}176 \\
(77.5)\end{array}$ & $\begin{array}{l}295 \\
(77.4)\end{array}$ \\
\hline & Unmarried & $4(2.6)$ & $\begin{array}{l}27 \\
(11.9)\end{array}$ & $\begin{array}{l}31 \\
(8.1)\end{array}$ \\
\hline & Divorced & $4(2.6)$ & $6(2.6)$ & $\begin{array}{l}10 \\
(2.62)\end{array}$ \\
\hline & Widow/Widowe & $\begin{array}{l}27 \\
17\end{array}$ & $\begin{array}{l}18 \\
(7.9)\end{array}$ & $\left.\mid \begin{array}{l}45 \\
(11.8)\end{array}\right)$ \\
\hline & Total & 154 & 227 & 381 \\
\hline & & $(100)$ & (100) & (100) \\
\hline
\end{tabular}

Table-2: Type family history, comorbidity, and stages of cancer patients.

\begin{tabular}{|c|c|c|c|c|}
\hline \multirow{22}{*}{$\begin{array}{l}\text { Disease and ICD } \\
10 \text { CODE }\end{array}$} & & $\begin{array}{l}\text { Male No. } \\
(\%)\end{array}$ & $\begin{array}{l}\text { Female No. } \\
(\%)\end{array}$ & $\begin{array}{l}\text { Total No. } \\
(\%)\end{array}$ \\
\hline & Mouth (6) & $2(1.3)$ & 0 & $2(.5)$ \\
\hline & Parotid gland (7) & 0 & $2(0.9)$ & $2(0.5)$ \\
\hline & Salivary glands (8) & $2(1.3)$ & 0 & $2(0.5)$ \\
\hline & Oropharynx (10) & $2(1.3)$ & 0 & $2(0.5)$ \\
\hline & Hypo pharynx (13) & $1(0.6)$ & $1(0.4)$ & $2(0.5)$ \\
\hline & Stomach (16) & $\begin{array}{l}18 \\
(11.7)\end{array}$ & $8(3.5)$ & $26(6.8)$ \\
\hline & Small intestine (17) & $3(1.9)$ & 0 & $3(0.8)$ \\
\hline & Colon (18) & $14(9.1)$ & $7(3.1)$ & $21(5.5)$ \\
\hline & $\begin{array}{l}\text { Recto sigmoid junction } \\
\text { (19) }\end{array}$ & $1(0.6)$ & $3(1.3)$ & $4(1.04)$ \\
\hline & Rectum (20) & 15 (9.7) & $13(5.7)$ & $28(7.3)$ \\
\hline & Anus/anal canal (21) & $1(0.6)$ & $1(0.4)$ & $2(0.5)$ \\
\hline & Liver /bile duct (22) & $\begin{array}{l}42 \\
(27.3)\end{array}$ & $14(6.2)$ & $56(14.7)$ \\
\hline & Gall bladder (23) & $2(1.3)$ & $6(2.5)$ & $8(2.09)$ \\
\hline & Biliary tract (24) & $7(4.5)$ & $4(1.8)$ & $11(2.8)$ \\
\hline & Bronchus/lungs (34) & 15 (9.7) & $18(7.9)$ & $33(8.7)$ \\
\hline & Urinary tract (64) & $1(0.6)$ & 0 & $1(0.2)$ \\
\hline & Bladder (67) & $2(1.3)$ & $1(0.4)$ & $3(0.7)$ \\
\hline & Thyroid (73) & $1(0.6)$ & $18(7.9)$ & $19(4.9)$ \\
\hline & Adrenal gland (74) & $1(0.6)$ & $1(0.4)$ & $2(0.5)$ \\
\hline & Parathyroid gland (75) & $2(1.3)$ & $2(1.9)$ & $4(1.04)$ \\
\hline & Breast (50) & $2(1.3)$ & $79(34.8)$ & $81(21.3)$ \\
\hline \multirow{3}{*}{ Family history } & Present & $\begin{array}{l}25 \\
(16.2)\end{array}$ & $29(12.8)$ & $\begin{array}{l}54 \\
(14.17)\end{array}$ \\
\hline & Absent & $\begin{array}{l}129 \\
(83.8)\end{array}$ & $198(87.2)$ & $\begin{array}{l}327 \\
(85.8)\end{array}$ \\
\hline & Total & $\begin{array}{l}154 \\
(100)\end{array}$ & $227(100)$ & $\begin{array}{l}381 \\
(100)\end{array}$ \\
\hline \multirow{8}{*}{ Comorbidity } & Hypertension & $\begin{array}{l}31 \\
(20.1)\end{array}$ & $28(12.3)$ & $59(15.4)$ \\
\hline & Diabetes mellitus & $\begin{array}{l}19 \\
(12.3)\end{array}$ & $17(7.5)$ & $36(9.44)$ \\
\hline & $\begin{array}{l}\text { Hypertension and } \\
\text { Diabetes mellitus }\end{array}$ & $\begin{array}{l}25 \\
(16.2)\end{array}$ & $17(7.5)$ & $\begin{array}{l}42 \\
(11.02)\end{array}$ \\
\hline & Asthma & $3(1.9)$ & $3(1.3)$ & $6(1.57)$ \\
\hline & Hypothyroidism & $1(1.6)$ & $2(.8)$ & $3(0.78)$ \\
\hline & Tuberculosis & $6(3.9)$ & $3(1.3)$ & $9(2.36)$ \\
\hline & No Disease & $\begin{array}{l}69 \\
(44.8)\end{array}$ & $157(69.2)$ & $\begin{array}{l}226 \\
(59.3)\end{array}$ \\
\hline & Total & $\begin{array}{l}154 \\
(100)\end{array}$ & $227(100)$ & $\begin{array}{l}381 \\
(100)\end{array}$ \\
\hline
\end{tabular}




\begin{tabular}{|l|l|l|l|l|}
\hline \multirow{4}{*}{ Stages of cancer presentation } & Stage 1 & $7(4.5)$ & $35(15.4)$ & $42(11.02)$ \\
\cline { 2 - 5 } & Stage 2 & $58(37.7)$ & $86(37.9)$ & $144(37.7)$ \\
\cline { 2 - 5 } & Stage 3 & $67(43.5)$ & $60(26.4)$ & $127(33.3)$ \\
\cline { 2 - 5 } & Stage 4 & $22(14.3)$ & $46(20.30$ & $68(17.84)$ \\
\cline { 2 - 5 } & Total & $154(100)$ & $227(100)$ & $381(100)$ \\
\hline
\end{tabular}

It was observed that breast cancer was the most common cancer followed by liver cancer and lung cancer and common cancers among the female group of population were cancer breast 79 (34.8\%) followed by cancer cervix 20 (8.8\%) followed by cancer ovary $19(8.4 \%)$ and thyroid 18 (7.9\%).

And the most common cancers among the male group of the population were cancer liver 42 (27.3\%) followed by cancer prostate 19 (12.3\%) followed by cancer stomach $18(11.7 \%)$ and lungs $15(9.7 \%)$.

The present study showed that around 69 (44.8\%) of them were free from any comorbidity followed by $31(20.1 \%)$ suffering from hypertension followed by $19(12.3 \%)$ with diabetes as comorbidity and 25 $(16.2 \%)$ suffered from both diabetes and hypertension.

It was observed that majority of cancer in males were diagnosed in stage III of disease accounting $67(43.5 \%)$ followed by stage II 58 (37.7\%) and in female patients majority of cancer was diagnosed in stage II 86 (37.9) followed by stage III 60 (26.4\%) (Table 2).

Table-3: Association of socio-demographic factors with breast cancer.

\begin{tabular}{|c|c|c|c|c|c|c|}
\hline \multirow{2}{*}{$\begin{array}{c}\text { Socio- } \\
\text { demographic } \\
\text { factors }\end{array}$} & \multicolumn{2}{|c|}{$\begin{array}{l}\text { Breast } \\
\text { cancer }\end{array}$} & \multirow[t]{2}{*}{$\begin{array}{l}\text { Odds } \\
\text { Ratio }\end{array}$} & \multicolumn{2}{|c|}{$\begin{array}{c}95 \% \text { Confidence } \\
\text { Intervals }\end{array}$} & \multirow[t]{2}{*}{$\begin{array}{l}x^{2}(p- \\
\text { value })\end{array}$} \\
\hline & $\begin{array}{l}\text { Presen } \\
\text { t No. }\end{array}$ & \begin{tabular}{|l|} 
Absent \\
No.
\end{tabular} & & \begin{tabular}{|l} 
Lower \\
Limit
\end{tabular} & $\begin{array}{l}\text { Upper } \\
\text { Limit }\end{array}$ & \\
\hline \multicolumn{7}{|c|}{ Age } \\
\hline$<50$ years & 43 & 99 & \multirow[t]{2}{*}{0.4353} & \multirow[t]{2}{*}{0.2644} & \multirow[t]{2}{*}{0.7164} & \multirow{2}{*}{$\begin{array}{l}11.01 \\
p=0.0009\end{array}$} \\
\hline$>50$ years & 38 & 201 & & & & \\
\hline \multicolumn{7}{|c|}{ Education } \\
\hline Illiterates & 19 & 104 & \multirow[t]{2}{*}{0.57} & \multirow[t]{2}{*}{0.3278} & \multirow[t]{2}{*}{1.0175} & \multirow{2}{*}{$\begin{array}{l}3.67 \\
p=0.055\end{array}$} \\
\hline Literates & 62 & 196 & & & & \\
\hline \multicolumn{7}{|c|}{ SES } \\
\hline Upper & 70 & 103 & \multirow[t]{2}{*}{12.17} & \multirow[t]{2}{*}{6.1729} & \multirow[t]{2}{*}{23.9983} & \multirow{2}{*}{$\begin{array}{l}69.80 \\
P<0.0001 *\end{array}$} \\
\hline Lower & 11 & 197 & & & & \\
\hline
\end{tabular}

A significant association was found between sociodemographic factors with breast cancer. It was found that in 19-50 years age group 43 (30.2\%) were diagnosed with breast cancer as compared to the older age group. This difference between age group with respect to cancer breast was found to be statistically significant $(p=0.0009)$, and $70(40.4 \%)$
Of the study subjects belonging to Upper SES were diagnosed with breast cancer when compared to lower SES $11(5.2 \%)$. This difference was found to be statistically significant $(p<0.01)$ (Table 3 ).

Table-4: Association of socio-demographic factors with cervical cancer.

\begin{tabular}{|c|c|c|c|c|c|c|}
\hline \multirow{2}{*}{$\begin{array}{c}\text { Socio- } \\
\text { demographic } \\
\text { factors }\end{array}$} & \multicolumn{2}{|c|}{$\begin{array}{l}\text { Cervical } \\
\text { cancer }\end{array}$} & \multirow[t]{2}{*}{$\begin{array}{l}\text { Odds } \\
\text { Ratio }\end{array}$} & \multicolumn{2}{|c|}{$\begin{array}{c}95 \% \text { Confidence } \\
\text { Intervals }\end{array}$} & \multirow[t]{2}{*}{$\begin{array}{l}x^{2}(p- \\
\text { value })\end{array}$} \\
\hline & $\begin{array}{l}\text { Present } \\
\text { No. }\end{array}$ & \begin{tabular}{|l|} 
Absent \\
No.
\end{tabular} & & $\begin{array}{l}\text { Lower } \\
\text { Limit }\end{array}$ & $\begin{array}{l}\text { Upper } \\
\text { Limit }\end{array}$ & \\
\hline \multicolumn{7}{|c|}{ Age } \\
\hline$<50$ years & 11 & 99 & \multirow[t]{2}{*}{1.33} & \multirow[t]{2}{*}{0.530} & \multirow[t]{2}{*}{3.353} & \multirow{2}{*}{$\begin{array}{l}0.38 \\
p=0.53\end{array}$} \\
\hline$>50$ years & 9 & 108 & & & & \\
\hline \multicolumn{7}{|c|}{ Education } \\
\hline Illiterates & 4 & 69 & \multirow[t]{2}{*}{0.5} & \multirow[t]{2}{*}{0.161} & \multirow[t]{2}{*}{1.5527} & \multirow{2}{*}{$p=0.316$} \\
\hline Literates & 16 & 138 & & & & \\
\hline \multicolumn{7}{|c|}{ SES } \\
\hline Upper & 3 & 110 & \multirow[t]{2}{*}{0.1556} & \multirow[t]{2}{*}{0.0443} & \multirow[t]{2}{*}{0.5472} & \multirow{2}{*}{$\begin{array}{l}P=0.001 \\
6 *\end{array}$} \\
\hline Lower & 17 & 97 & & & & \\
\hline
\end{tabular}

Women with lower SES had a higher risk of cancer cervix $17(14.9 \%)$ compared to higher SES 3(2.6\%). This difference was found to be statistically significant $(p=0.0016)$ (Table 4$)$.

Table-5: Association of socio-demographic factors with lung cancer.

\begin{tabular}{|c|c|c|c|c|c|c|}
\hline \multirow{2}{*}{$\begin{array}{c}\text { Socio- } \\
\text { demographic } \\
\text { factors }\end{array}$} & \multicolumn{2}{|c|}{ Lung cancer } & \multirow[t]{2}{*}{$\begin{array}{l}\text { Odds } \\
\text { Ratio }\end{array}$} & \multicolumn{2}{|c|}{$\begin{array}{l}95 \% \text { Confidence } \\
\text { Intervals }\end{array}$} & \multirow[t]{2}{*}{$\begin{array}{l}x^{2}(p- \\
\text { value })\end{array}$} \\
\hline & \multirow[t]{2}{*}{$\begin{array}{l}\text { Present } \\
\text { No. }\end{array}$} & \begin{tabular}{|l|} 
Absent \\
No.
\end{tabular} & & $\begin{array}{l}\text { Lower } \\
\text { Limit }\end{array}$ & $\begin{array}{l}\text { Upper } \\
\text { Limit }\end{array}$ & \\
\hline \multicolumn{6}{|c|}{ Age } & \\
\hline$<50$ years & 13 & 129 & \multirow[t]{2}{*}{1.1035} & \multirow[t]{2}{*}{0.5311} & \multirow[t]{2}{*}{2.293} & \multirow{2}{*}{$\begin{array}{l}0.07 \\
p=0.79\end{array}$} \\
\hline$>50$ years & 20 & 219 & & & & \\
\hline \multicolumn{7}{|c|}{ Sex } \\
\hline Male & 15 & 139 & \multirow[t]{2}{*}{1.253} & \multirow[t]{2}{*}{0.611} & \multirow[t]{2}{*}{2.569} & 0.38 \\
\hline Female & 18 & 209 & & & & $p=0.537$ \\
\hline \multicolumn{7}{|c|}{ Education } \\
\hline Illiterates & 4 & 119 & \multirow[t]{2}{*}{0.2654} & \multirow[t]{2}{*}{0.0912} & \multirow[t]{2}{*}{0.7728} & \multirow[t]{2}{*}{$P=0.01 *$} \\
\hline Literates & 29 & 229 & & & & \\
\hline \multicolumn{7}{|c|}{ SES } \\
\hline Upper & 16 & 157 & \multirow[t]{2}{*}{1.145} & \multirow[t]{2}{*}{0.5603} & \multirow[t]{2}{*}{2.339} & \multirow{2}{*}{$\begin{array}{l}0.14 \\
p=0.708\end{array}$} \\
\hline Lower & 17 & 191 & & & & \\
\hline
\end{tabular}

Lung cancer was seen more in literates when compared to illiterates. In the present study, Lung cancer was seen in $29(11.2 \%)$ of literates while $4(3.3 \%)$ in illiterates.

This difference between education with respect to Lung cancer was found to be statistically significant with $p$-value $(p=0.01)$ (Table 5). 
Table-6: Association of socio-demographic factors with liver cancer.

\begin{tabular}{|c|c|c|c|c|c|c|}
\hline \multirow{2}{*}{$\begin{array}{l}\text { Socio- } \\
\text { demographic } \\
\text { factors }\end{array}$} & \multicolumn{2}{|c|}{ | Liver cancer } & \multirow[t]{2}{*}{$\begin{array}{l}\text { Odds } \\
\text { Ratio }\end{array}$} & \multicolumn{2}{|c|}{$\begin{array}{c}95 \% \text { Confidence } \\
\text { Intervals }\end{array}$} & \multirow[t]{2}{*}{$\begin{array}{l}x^{2}(p- \\
\text { value })\end{array}$} \\
\hline & $\begin{array}{l}\text { Present } \\
\text { No. }\end{array}$ & $\begin{array}{l}\text { Absent } \\
\text { No. }\end{array}$ & & $\begin{array}{l}\text { Lower } \\
\text { Limit }\end{array}$ & $\begin{array}{l}\text { Upper } \\
\text { Limit }\end{array}$ & \\
\hline \multicolumn{7}{|c|}{ Age } \\
\hline$<50$ years & 11 & 131 & \multirow[t]{2}{*}{0.362} & \multirow[t]{2}{*}{0.1806} & \multirow[t]{2}{*}{0.7257} & \multirow{2}{*}{$\begin{array}{l}8.73, \\
p=0.003 *\end{array}$} \\
\hline$>50$ years & 45 & 194 & & & & \\
\hline \multicolumn{7}{|c|}{ Sex } \\
\hline Male & 42 & 112 & \multirow[t]{2}{*}{5.7054} & \multirow[t]{2}{*}{2.988} & \multirow[t]{2}{*}{10.892} & 32.6 \\
\hline Female & 14 & 213 & & & & $p=0.000$ \\
\hline \multicolumn{7}{|c|}{ Education } \\
\hline Illiterates & 24 & 99 & \multirow[t]{2}{*}{1.712} & \multirow[t]{2}{*}{0.959} & \multirow[t]{2}{*}{3.056} & \multirow{2}{*}{$\begin{array}{l}3.36 \\
p=0.066\end{array}$} \\
\hline Literates & 32 & 226 & & & & \\
\hline \multicolumn{7}{|c|}{ SES } \\
\hline Upper & 17 & 156 & \multirow[t]{2}{*}{0.472} & \multirow[t]{2}{*}{0.256} & \multirow[t]{2}{*}{0.868} & \multirow{2}{*}{$\begin{array}{l}6, \\
p=0.014 *\end{array}$} \\
\hline Lower & 39 & 169 & & & & \\
\hline
\end{tabular}

When compared socio-demographic factors with cancer liver. It was found that study subjects in the age group $>50$ years i.e. $45(18.8 \%)$ had more diagnosed lung cancer as compared to $<50$ years age group. This difference between the age group with respect to cancer liver was found to be statistically significant $(p=0.00313)$. Liver cancer was different in males when compared to females.

In males, $42(27.2 \%)$ had liver cancer and 14 $(6.1 \%)$ were seen in females. This difference between gender with respect to liver cancer was found to be statistically significant $(p=<0.0001)$. Liver cancer was seen more in Lower SES i.e. 39 $(18.7 \%)$ when compared to Higher SES i.e. $17(9.8 \%)$ of study subjects. This difference was found to be statistically significant $(p=0.014)$ (Table $6)$.

Cancer breast $(21.3 \%)$ in subjects was most prevalent, followed by the liver and biliary tract $(14.7 \%)$, followed by lungs $(8.7 \%)$ a study done by Puri et al, showed that Cancer lung $(22.3 \%)$ in subjects was most prevalent, followed by ca breast (13.1\%) [6].

A study done in Kashmir by Wani et al shows that the ten leading sites of cancer are esophagus and GE Junction, lung, stomach, colorectal, lymphomas, skin, laryngopharynx, acute leukemias, prostate and brain in males. In females, the leading sites are breast, esophagus, and GE Junction, ovary, colorectal, stomach, lung, gallbladder, lymphomas, acute leukemias and brain.
$27 \%$

\section{Discussion}

The esophagus and stomach alone constituted over of the total cancers, these findings were not similar to the present study [7]. Another study in Maharashtra by Bangal et al showed that the Cancer cervix was the commonest site $(19.5 \%)$, followed by cancer breast $(10.2 \%)$, cancer of the lung $(9.7 \%)$ and cancer of the floor of mouth $(7.3 \%)$.

In males, the commonest site for cancer was lung $(9.7 \%)$, followed by the floor of mouth $(7.3 \%)$, while in females; cancer cervix (19.5\%) predominated, followed by cancer breast $(10.2 \%)$ and cancer ovary (2.4\%) [8].

Similar study in Kolar by Kalyani et al showed that Cancer of oral cavity predominated in both genders and the top ten sites most frequently involved by cancer in males were oral cavity, stomach, esophagus, bone, NHL, prostate, liver, larynx, penis, and Hodgkin's disease/bladder cancer, whereas, the sites in females were oral cavity, cervix, breast, stomach, esophagus, thyroid, ovary, bone, rectum, and melanoma skin, this result was contrasted to present study [9].

In the Global Adult Tobacco Survey 2009-10 (GATS), the majority of study subjects were homemakers and self-employed and this finding was similar to the present study [10].

Similar observations were found in a study done in Karnataka by Rajesh et al, shows that $23.3 \%$ were unemployed and unskilled $46.6 \%$ [11].

A study done by Suthahar et al, at Malaysia findings were similar to the present study, which shows that $50 \%$ were unemployed and $64.7 \%$ was pensioner [12].

A study done by Kaku et al, at south India findings were similar to the present study, $22 \%$ were illiterate and 33\% were literate by occupation [13].

According to National cancer registry program under Indian council of medical research Consolidated report of Population-based cancer registries, Bangalore reports that Males: The leading sites of cancer were hypopharynx (8.4\%), esophagus $(7.9 \%)$, lung $(7.0 \%)$, tongue $(6.7 \%)$ and mouth $(6.5 \%)$ followed by others, the leading cancer sites among females were cancer of the cervix, accounting for about $27.3 \%$ of cancers followed by breast $(15.6 \%)$, mouth $(10.3 \%)$, ovary $(5.9 \%)$ and 
Esophagus (5.1\%) [14]

In the present study, $70(40.4 \%)$ of the study subjects belonging to Upper SES were diagnosed with breast cancer when compared to lower SES $11(5.2 \%)$. This difference was found to be statistically significant $(p<0.01)$.

A study was done in Delhi by Kaur et al .showed that there was no significant difference in literacy status and Ca breast[15].

Neal and Allgar's study showed that there was a significant difference in age group and diagnosis of Ca breast [16].

Cervical cancer was seen more in lower SES i.e. $17(14.9 \%)$ when compared to higher SES 3(2.6\%). This difference was found to be statistically significant $(p=0.0016)$.

Thulaseedharan et al study showed that the risk significantly increased with increasing age. There was a 2.5 -fold $(95 \% \mathrm{CI}=1.59-3.77)$ increase in risk among women aged 50-59 years compared to those aged 30-39, and a significant dose-response relationship ( $p$-value $<0.001$ ) was also observed. This was not similar to the present study[17].

\section{Limitation}

This study is a hospital-based study so it cannot generalize to the population.

- As the study was done for 1 year, so the exact pattern of cancers prevalent in the region and neighboring areas couldn't be estimated.

- Complete data from patients pertaining to follow up and the outcome couldn't be retrieved owing to the study period being Less.

\section{Conclusion}

Breast cancer more common among younger age groups and upper SES, Cervical cancer more common among lower SES, Lung cancer in literates' Liver cancer more common among older age group and lower SES. So, these groups can be targeted for the population for health education and screening for early diagnosis and treatment.

\section{What does the study add to the existing knowledge}

In the present study, it was found that the majority of patients were reporting in stage III of cancer and $14 \%$ shows positive family history.
- The majority of patients were reporting in stage III CA so health education regarding early diagnosis and treatment recommended.

- Clinicians should elicit family histories and counsel about health-related behavior's

- In the present study, a statistically significant association was found between sociodemographic factors and types of cancers, so those groups can be targeted for health education and screening for early diagnosis and treatment.

- Breast cancer more common among younger age group and upper SES

- Cervical cancer more common among lower SES

- Lung cancer in literates

- Liver cancer more common among older age group and lower SES

\section{Author's contribution}

Both the authors, Dr. Antoney Unni Xavier and Dr. Amgiasvasanth A M contributed equally in the conduct of the study, data analysis and in the preparation of the manuscript.

\section{Reference}

01. Cancer in Developing Countries, INCTR International Network for Cancer Treatment and Research [Internet].

Available from: [Article] [Crossref]

02. Fact Sheets by Cancer [Internet]. [cited 2015 Mar 16].

Available from: [Article] [Crossref]

03. Mallath MK, Taylor DG, Badwe RA, Rath GK, Shanta V, Pramesh CS, et al. The growing burden of cancer in India: epidemiology and social context. Lancet Oncol. 2014;15(6)e205e212.

doi: [Article] [Crossref]

04. Statistics of Breast Cancer in India. Global Comparison.

Available from: [Article] [Crossref]

05. Khanna A. Current Status of Cancer BurdenGlobal and Indian Scenario. Biomed Res J. 2014;1(1)1-5.

[Crossref] 
06. Ashat M, Goel N, Puri S, Pandey A, Singh A, Kaushal V. Socio-demographic characteristics of cancer patients- Hospital based cancer registry in a tertiary care hospital of India. Indian J Cancer. 2014;51(1)1-4.

doi: [Article] [Crossref]

07. Wani M, Jan F, Khan N, Pandita K, Khurshid R, Khan S. Cancer trends in Kashmir; common types, site incidence and demographic profilesNational Cancer Registry 2000-2012. Indian J Cancer. 2014;51(2)133-137. doi: [Article] [Crossref]

08. Bangal R, Giri P, Bangal S, More M, Singh K. Socio-demographic profile and associated risk factors in cancer patients attending the Oncology OPD of a tertiary care teaching hospital in Western Maharashtra, India. Int J Med Sci Public Heal. 2014;3(12)1.

[Crossref]

09. Kalyani R, Das S, Singh B, Kumar H. Cancer profile in the department of pathology of sri devaraj urs medical college, Kolar- A ten years study. Indian J Cancer. 2010;47(2)160-165. doi: [Article] [Crossref]

10. World Health Organization. Publications, The Global Adult Tobacco Survey (GATS) India. WHO. 2009-2010.

Available at [Article] [Crossref]

11. Rajesh N, Sreelakshmi K, Ramesh K. Profile of oral cancer patients attending tertiary care hospital, Bellary, Karnataka, India. Int J Curr Res Aca Rev. 2014;2(8)46-52.

[Crossref]
12. Suthahar A, Gurpreet $K$, Ambigga D, Maniam T, Dhachayani S, Fuad I, et al. A profile of cancer patient outcomes from a tertiary care teaching hospital in Malaysia. Singapore Med J. $2009 ; 50(7) 720-723$.

[Crossref]

13. Kaku M, Mathew A, Rajan B. Impact of socioeconomic factors in delayed reporting and latestage presentation among patients with cervix cancer in a major cancer hospital in South India. Asian Pac J Cancer Prev. 2008;9(4)589-594. [Crossref]

14. NCRP. Three-Year Report of Population Based Cancer Registries 2009-2011. National Cancer Registry Programme (Indian Council of Medical Research), Bangalore. 2013. 2015 [Article] [Crossref]

15. Kaur N, Attam A, Saha S, Bhargava SK. Breast Cancer Risk Factor Profile in Indian Women. JIMSA. 2011;24(4)163-165.

[Crossref]

16. Neal RD, Allgar VL. Sociodemographic factors and delays in the diagnosis of six cancersanalysis of data from the "National Survey of NHS Patients- Cancer". $\mathrm{Br} J$ Cancer. 2005;92(11)1971-1975. doi: [Article] [Crossref]

17. Thulaseedharan J V, Malila N, Hakama M, Esmy PO, Cheriyan M, Swaminathan $R$, et al. Socio Demographic and Reproductive Risk Factors for Cervical Cancer - a Large Prospective Cohort Study from Rural India. Asian Pacific J Cancer Prev. 2012;13(6)2991-2995. doi: [Article] [Crossref] 